\title{
Determination of Growth Parameters of Methylamine-using Bacteria
}

\author{
By J. D. LEGAN† AND J. D. OWENS* \\ Department of Food Technology, University of Reading, Whiteknights, Reading RG6 2AP, UK
}

(Received 19 June 1986; revised 23 October 1986)

Growth parameters on methylamine and glucose were determined for obligately methylotrophic bacterium B6/2, facultatively methylotrophic bacterium DT26 and Pseudomonas NB4, which is able to use methylamine as sole nitrogen source but not as sole carbon and energy source. Substrate saturation constants were determined by a respirometric method. Values obtained for maximum specific growth rates $\left(\mathrm{h}^{-1}\right)$, substrate saturation constants $\left(\mu \mathrm{mol} \mathrm{l}^{-1}\right)$, yield coefficient as $50 \%$ maximum growth rate [( $\mathrm{g}$ dry biomass) $\left.\mathrm{mol}^{-1}\right]$ and maintenance coefficient [mmol (g dry biomass) $)^{-1} \mathrm{~h}^{-1}$, respectively, were $0.33,36,21 \cdot 2$ and $3 \cdot 2$ for $\mathrm{B} 6 / 2$ with methylamine as sole carbon, energy and nitrogen source, $0.09,5 \cdot 1,15 \cdot 6$ and 1.8 for DT26 with methylamine as sole carbon, energy and nitrogen source, $0 \cdot 20,2 \cdot 5,94$ and $0 \cdot 70$ for DT26 with glucose as sole carbon and energy source and $\mathrm{NH}_{4}^{+}$as sole nitrogen source, and $0.58,6 \cdot 1,67$ and 0.63 for NB4 with glucose as sole carbon and energy source and $\mathrm{NH}_{4}^{+}$as sole nitrogen source. Pseudomonas NB4 grew with a maximum specific growth rate of 0.35 with glucose as sole carbon and energy source and methylamine as sole nitrogen source.

\section{INTRODUCTION}

Bacteria able to use methylamines in aerobic environments include specialist, obligately methylotrophic bacteria able to use only $C_{1}$-compounds as sole carbon and energy sources, generalist, facultatively methylotrophic bacteria able to use $C_{1}$-compounds or non- $C_{1}$ compounds as sole carbon and energy sources, and methazotrophic bacteria able to use methylamines as sole nitrogen sources but not as sole carbon and energy sources (Anthony, 1982). In order to study, both by computer simulations and in laboratory mixed continuous cultures, the competition by these three types of bacteria for methylamine it was first necessary to determine the growth parameters of the selected strains. Maximum specific growth rates, substrate saturation constants, yield coefficients and maintenance coefficients on methylamine and glucose were determined. The determination of substrate saturation constants by the direct assay of nutrient concentrations in steady-state continuous cultures is often difficult or impossible due to the low concentrations present and we, therefore, investigated Harrison's (1973) respirometric method.

\section{METHODS}

Bacterial strains. The strains used were obligate methylotroph B6/2 (Legan \& Owens, 1985; Legan et al., 1987), facultative methylotroph DT26 (Legan et al., 1987) and methazotroph NB4 (Bicknell \& Owens, 1980). Strain NB4 is a Pseudomonas species able to use as sole nitrogen sources, but not as sole carbon sources, methylamine, dimethylamine, trimethylamine, trimethylamine oxide and betaine.

Culture media. These were as used by Legan et al. (1987).

Continuous culture apparatus. This was similar to that described by Legan et al. (1987) except that only one medium reservoir was used and cultures were grown with a single carbon source.

Determination of maximum specific growth rates. Maximum specific growth rates were measured by the washout

† Present address: Flour Milling and Baking Research Association, Chorleywood, Rickmansworth, Herts. WD3 SSH, UK. 
from continuous culture method of Pirt \& Callow (1960). Washout was measured as change in culture optical density at $450 \mathrm{~nm}$ against a distilled water blank in a Pye-Unicam SP6-350 spectrophotometer.

Determination of substrate saturation constants by respirometry. The method used was similar to that of Harrison (1973) with the difference that culture samples from carbon and energy source-limited continuous cultures growing at growth rates greater than $70 \%$ of maximum were used directly in the respirometer without dilution in nitrogenand carbon-free diluent. Hence, all nutrients, other than the carbon and energy source, were present and the microbes were therefore able to grow upon the addition of carbon and energy source during the measurements of respiration rates. Since the Monod substrate saturation constant relates to microbial growth, this latter condition is an important one if respirometric methods are to be used to attempt to measure substrate saturation constants for growth. Samples were taken from relatively fast-growing cultures so that the concentrations of nutrient they were exposed to in the respirometer were of the same order or less than those previously experienced in the culture.

For a culture growing on a single carbon and energy source, at a particular moment (Pirt, 1975):

Total rate of oxygen $=$ rate of consumption for + rate of consumption for + rate of consumption for consumption generation of energy for growth maintenance raising oxidation level of carbon source to that of biomass for assimilation

That is

$$
q_{\mathrm{O}_{2}}^{\mathrm{T}} x=q_{\mathrm{O}_{2}}^{\mathrm{EG}} x+q_{\mathrm{O}_{2}}^{\mathrm{EM}} x+q_{\mathrm{O}_{2}}^{\mathrm{C}} x
$$

where $q_{\mathrm{O}_{2}}$ are the relevant metabolic quotients for oxygen consumption [mol oxygen (g dry biomass)-1 $\mathrm{h}^{-1}$ ] and $x$ is the concentration of biomass ( $\mathrm{g}$ dry biomass $\mathrm{1}^{-1}$ ). For carbon sources at an oxidation level similar to that of biomass, $q_{\mathrm{O}_{2}}^{\mathrm{C}}$ approximates to zero.

Under constant conditions, the number of moles of oxygen required to oxidize one mole of energy source is a constant, $k_{1}$, and:

$$
q_{\mathrm{O}_{2}}^{\mathrm{EG}}=k_{1} q_{\mathrm{S}}^{\mathrm{EG}}
$$

where $q_{\mathrm{S}}^{\mathrm{EG}}$ is the metabolic quotient for the consumption of energy source for the generation of energy for growth.

Under constant conditions, the amount of substrate assimilated, and the amount of oxygen used in its oxidation to the oxidation level of biomass, bears a constant relationship to the amount of substrate oxidized for energy for growth, such that:

$$
q_{\mathrm{O}_{2}}^{\mathrm{C}}=k_{2} q_{\mathrm{S}}^{\mathrm{EG}}
$$

where $k_{2}$ is a constant. Thus

$$
q_{\mathrm{O}_{2}}^{\mathrm{EG}}+q_{\mathrm{O}_{2}}^{\mathrm{C}}=k_{1} q_{\mathrm{S}}^{\mathrm{EG}}+k_{2} q_{\mathrm{S}}^{\mathrm{EG}}
$$

The rate of consumption of energy source for the generation of energy for growth is also given by $\mu x / Y_{\mathrm{S}}^{\mathrm{EG}}$, where $\mu$ is the specific growth rate $\left(\mathrm{h}^{-1}\right)$ and $Y_{\mathrm{S}}^{\mathrm{EG}}$ is the yield coefficient [g dry biomass (mol substrate) ${ }^{-1}$ ] for use of the energy source for growth.

$$
\begin{aligned}
q_{\mathrm{O}_{2}}^{\mathrm{EG}}+q_{\mathrm{O}_{2}}^{\mathrm{C}} & =\mu k_{1} / Y_{\mathrm{S}}^{\mathrm{EG}}+\mu k_{2} / Y_{\mathrm{S}}^{\mathrm{EG}} \\
& =\mu\left(k_{1}+k_{2}\right) / Y_{\mathrm{S}}^{\mathrm{EG}}
\end{aligned}
$$

Since $Y_{\mathrm{S}}^{\mathrm{EG}}$ is a constant, this predicts that the rate of oxygen consumption for substrate oxidation for energy for growth and assimilation is directly proportional to the specific growth rate. This has been shown to be true for Klebsiella aerogenes ( $K$. pneumoniae) grown in carbon-limited and carbon-excess conditions (Neijssel \& Tempest, 1976). $\left(q_{\mathrm{O}_{2}}^{\mathrm{EC}}+q_{\mathrm{O}_{2}}^{\mathrm{C}}\right)$, or $q_{\mathrm{O}_{2}}^{\mathrm{EG}}$ for substrates at the same oxidation level as biomass, may, therefore, be used to assess the effects of substrate concentration on growth rate in the determination of Monod substrate saturation constants for growth. Since

$$
q_{\mathrm{O}_{2}}^{\mathrm{EG}}+q_{\mathrm{O}_{2}}^{\mathrm{C}}=q_{\mathrm{O}_{2}}^{\mathrm{T}}-q_{\mathrm{O}_{2}}^{\mathrm{EM}}
$$

$\left(q_{\mathrm{O}_{2}}^{\mathrm{EG}}+q_{\mathrm{O}_{2}}^{\mathrm{C}}\right)$ can be determined if it is assumed that the rate of oxygen consumption for maintenance is the same as the endogenous respiration rate measured in the absence of added substrate.

A Rank oxygen electrode respirometer was assembled using molar $\mathrm{KCl}$ as electrolyte and the platinum cathode was polarized at $-0.6 \mathrm{~V}$ relative to the silver anode. The temperature of the respirometer chamber was maintained at $25^{\circ} \mathrm{C}$ by circulating water through the chamber jacket. A few crystals of sodium dithionite were 
added and the chamber stopper was inserted. The chart recorder pen-offset was adjusted so that the residual current with sodium dithionite present was recorded as zero. The electrode output fell from $100 \%$ to $10 \%$ in less than $5 \mathrm{~s}$ upon the addition of dithionite. Hence, delay in electrode response introduced negligible error in the measurements of respiration rate.

A range of glucose and methylamine solutions from 0.5 to $50 \mathrm{mmol} \mathrm{l}^{-1}$ was prepared $24 \mathrm{~h}$ or less before use, stored at $4-6{ }^{\circ} \mathrm{C}$ and warmed to room temperature $\left(21-29^{\circ} \mathrm{C}\right)$ when required.

A $5 \mathrm{ml}$ sample from a steady-state chemostat culture, growing at $70 \%$ or more of its maximum rate, was pipetted into the electrode chamber and stirred vigorously until the maximum dissolved oxygen tension was reached. The electrode output was adjusted to $8 \mathrm{mV}$. The chamber stopper was adjusted to exclude all air bubbles and the endogenous respiration rate was measured (fall in electrode output/time, in the absence of substrate). Ten microlitres of the least concentrated substrate solution was added to the culture through the hole in the chamber stopper and the initial respiration rate was noted (fall in electrode output/time, immediately after addition of substrate). When the rate had returned to the endogenous value the procedure was repeated.

Duplicate $10 \mu \mathrm{l}$ volumes of the other substrate solutions were added to the culture in order of increasing concentration. Substrate concentrations presented to the culture were in the range $1-100 \mu \mathrm{mol} \mathrm{I}^{-1}$. When the dissolved oxygen tension in the respirometer fell below $50 \%$ of saturation the culture was re-aerated before addition of further substrate. The period over which determinations were made on a culture sample was restricted to less than about $10 \%$ of the minimum population doubling time in order to limit the change in biomass concentration during a series of measurements.

The rate of oxygen consumption for energy generation for growth and for substrate assimilation $\left(q_{\mathrm{O}_{2}}^{\mathrm{EG}} x+q_{\mathrm{O}_{2}}^{\mathrm{C}} x\right)$ was calculated by subtracting the endogenous respiration rate from the initial total respiration rate $\left(q_{\mathrm{O}_{2}}^{\mathrm{T}} x-q_{\mathrm{O}_{2}}^{\mathrm{EE}} x\right)$ and $K_{\mathrm{s}}$ was determined from a plot of $\left[1 /\left(q_{\mathrm{O}_{2}}^{\mathrm{T}} x-q_{\mathrm{O}_{2}}^{\mathrm{EE}} x\right)\right]$ versus (1/substrate concentration).

Determination of substrate saturation constant by assay of glucose concentrations in steady-state continuous cultures. This was done only for strain NB4. A sample of culture was removed from the chemostat and rapidly filtered through a membrane filter (Oxoid N25/22UP, $25 \mathrm{~mm}$ diameter, $0.22 \mu \mathrm{m}$ pore size) held in a Millipore Swinnex filter holder. Two millilitres of culture filtrate could be obtained in less than $10 \mathrm{~s}$. Two millilitres of filtrate were evaporated to dryness at $50^{\circ} \mathrm{C}$ under vacuum and redissolved in $0.2 \mathrm{ml}$ distilled water. Glucose was assayed using a GOD-Perid test kit (Boehringer) and measuring absorbance at $436 \mathrm{~nm}$ in a Pye-Unicam SP6 350 spectrophotometer fitted with a $4 \mathrm{~cm}$ cuvette. With this technique concentrations of glucose in the range 10 $50 \mu \mathrm{mol}^{-1}$ and upwards could be determined.

The substrate saturation constant was calculated using the Monod equation, $K_{\mathrm{s}}=s\left(\mu_{\mathrm{m}}-\mu\right) / \mu$, where $s$ is the concentration of growth-rate-limiting glucose, $\mu_{\mathrm{m}}$ is the maximum specific growth rate, and $\mu$ is the specific growth rate and equal to the dilution rate in the steady-state chemostat culture.

Determination of yield and maintenance coefficients. Organisms were grown in continuous culture and allowed to reach steady state at a number of growth rates between $20 \%$ and $80 \% \mu_{\mathrm{m}}$. At each growth rate, biomass concentrations of two samples from the culture were determined and the mean value found. Graphs were plotted of (1/yield) against (1/growth rate) (Pirt, 1975). The slope gave the maintenance coefficient, and the yield coefficient, at a relative growth rate of $50 \% \mu_{\mathrm{m}}$, was read from the graph.

Biomass concentrations in cultures were determined by filtering culture samples through weighed, dried membrane filters (Millipore HA $47 \mathrm{~mm}$ diameter, $0.45 \mu \mathrm{m}$ pore size) under pressure (up to $1000 \mathrm{kPa}$ ) in a Sartorius pressure filtration cell (SM16224). Filters were dried at $105^{\circ} \mathrm{C}$ and reweighed. Cells on filters were not rinsed with water since it was estimated that the amount of retained salts was negligible, but weights were corrected for materials leached from the filters.

Ten replicate determinations using $25 \mathrm{ml}$ samples from cultures of strain DT26 (320 mg dry biomass $\left.\mathrm{l}^{-1}\right)$ and strain NB4 (220 and $280 \mathrm{mg}$ dry biomass $\mathrm{I}^{-1}$ ) yielded coefficients of variation (standard deviation $\left.\times 100 / \mathrm{mean}\right)$ of $3-7 \%$.

\section{RESULTS AND DISCUSSION}

Determination of maximum specific growth rates by washout from continuous culture

The maximum specific growth rates determined are shown in Table 1. As expected, obligate methylotroph B6/2 grew faster $\left(\mu_{\mathrm{m}} 0.33 \mathrm{~h}^{-1}\right)$ than facultative methylotroph DT26 $\left(\mu_{\mathrm{m}} 0.09 \mathrm{~h}^{-1}\right)$ when methylamine was the sole source of carbon, energy and nitrogen. Hence, in environments with a continuous, relatively high concentration of methylamine the specialist, obligate methylotroph can be expected to outcompete and exclude the generalist, facultative methylotroph.

Also, as might be anticipated, facultative methylotroph DT26 grew faster $\left(\mu_{\mathrm{m}} 0 \cdot 20 \mathrm{~h}^{-1}\right)$ on glucose/ammonium than on methylamine $\left(\mu_{\mathrm{m}} 0.09 \mathrm{~h}^{-1}\right)$. 
Table 1. Growth parameters of obligate methylotroph B6/2, facultative methylotroph DT26 and methazotroph NB4

\begin{tabular}{|c|c|c|c|c|c|}
\hline \multirow[b]{3}{*}{ Strain ... } & \multicolumn{5}{|c|}{ Growth substrate } \\
\hline & \multicolumn{2}{|c|}{ Methylamine } & \multicolumn{2}{|c|}{ Glucose/ $/ \mathbf{N H}_{4}$} & \multirow{2}{*}{$\begin{array}{l}\text { Glucose/ } \\
\text { methylamine } \\
\text { NB4 }\end{array}$} \\
\hline & B6/2 & DT26 & DT26 & NB4 & \\
\hline $\begin{array}{l}\text { Mean maximum specific growth } \\
\text { rate }\left(h^{-1}\right)\end{array}$ & $0 \cdot 33$ & 0.09 & $0 \cdot 20$ & 0.58 & $0 \cdot 35^{*}$ \\
\hline No. of determinations & 5 & 7 & 5 & 5 & 3 \\
\hline Coefficient of variation $(\%) \dagger$ & 5.9 & $10 \cdot 4$ & $6 \cdot 3$ & $7 \cdot 6$ & $6 \cdot 7$ \\
\hline $\begin{array}{l}\text { Substrate saturation constant for } \\
\text { carbon and energy source }\left(\mu \mathrm{mol} \mathrm{l}^{-1}\right)\end{array}$ & $36 \ddagger$ & $5 \cdot 1 \ddagger$ & $2 \cdot 5 \ddagger$ & $6 \cdot 1 \ddagger(5 \cdot 9) \S$ & ND \\
\hline No. of determinations & 4 & 6 & 4 & $4(6)$ & \\
\hline Coefficient of variation $(\%) \dagger$ & 12 & 24 & 14 & $49(28)$ & \\
\hline $\begin{array}{l}\text { Yield on carbon and energy source } \\
\text { at } 50 \% \text { maximum growth rate } \\
{\left[(\mathrm{g} \text { dry biomass }) \mathrm{mol}^{-1}\right]}\end{array}$ & $21 \cdot 2$ & $15 \cdot 6$ & $94 \|$ & $67 \|$ & ND \\
\hline $\begin{array}{l}\text { Maintenance coefficient on carbon } \\
\text { and energy source [mmol ( } \mathrm{g} \text { dry } \\
\text { biomass) }{ }^{-1} \mathrm{~h}^{-1} \text { ] }\end{array}$ & $3 \cdot 2$ & 1.8 & $0 \cdot 70 \|$ & $0.63 \|$ & ND \\
\hline \multicolumn{6}{|c|}{$\begin{array}{l}\text { ND. Not determined. } \\
\text { Growth rate of culture limited by nitrogen source (methylamine) prior to determination of maximum growth } \\
\text { rate. All other cultures were carbon limited prior to determination of maximum growth rate. } \\
+ \text { Standard deviation } \times 100 / \text { mean. } \\
\ddagger \text { Determined by respirometry. } \\
\S \text { Determined by direct assay of glucose concentrations in steady-state continuous cultures at dilution rates from } \\
0.30 \text { to } 0.53 \mathrm{~h}^{-1} \text {. }\end{array}$} \\
\hline
\end{tabular}

Methazotroph NB4 grew faster both on glucose/ammonium $\left(\mu_{\mathrm{m}} 0.58 \mathrm{~h}^{-1}\right)$ and on glucose/methylamine $\left(\mu_{\mathrm{m}} 0.35 \mathrm{~h}^{-1}\right)$ than did strain DT26 on glucose/ammonium $\left(\mu_{\mathrm{m}} 0.20 \mathrm{~h}^{-1}\right)$ or on methylamine $\left(\mu_{\mathrm{m}} 0.09 \mathrm{~h}^{-1}\right)$. Hence, in constant environments with high concentrations of glucose/ammonium or of glucose/methylamine, strain NB4 would outgrow and exclude strain DT26.

\section{Determination of substrate saturation constants}

An example of a chart record of oxygen uptake is shown in Fig. 1 and of a double-reciprocal plot used to obtain $K_{\mathrm{s}}$ values from oxygen electrode data in Fig. 2. The $K_{\mathrm{s}}$ values for all strains are given in Table 1, including one determination by the direct assay of glucose concentrations in steady-state chemostat cultures of strain NB4. This latter result was very close to that obtained by the respirometric technique and lends support to the validity of determining substrate saturation constants of growing cultures by respirometry.

Plots of growth rate versus concentration of growth-rate-limiting nutrient, using the Monod equation, for strains B6/2 and DT26 on methylamine and for strains DT26 and NB4 on glucose are shown in Figs 3 and 4 respectively. The curves for strains B6/2 and DT 26 cross at a growth rate of $0.05 \mathrm{~h}^{-1}$. Hence, in methylamine-limited chemostat cultures with a constant nutrient supply and operated at dilution rates below this value, facultative methylotroph DT26 will grow faster than obligate methylotroph B6/2 and exclude it from a mixed culture. At all dilution rates above $0.05 \mathrm{~h}^{-1}$ strain B6/2 will exclude strain DT26. Methazotroph NB4 grew faster than facultative methylotroph DT26 at all concentrations of glucose (Fig. 4) and will, therefore, outgrow and exclude strain DT26 at all dilution rates in glucose-limited chemostat cultures with a constant nutrient supply.

\section{Determination of yield and maintenance coefficients}

An example plot of (1/yield) against (1/growth rate) is shown in Fig. 5, and the yield and maintenance coefficients determined are given in Table 1 . The yield coefficients $(15.6$ and $21 \cdot 2 \mathrm{~g}$ 


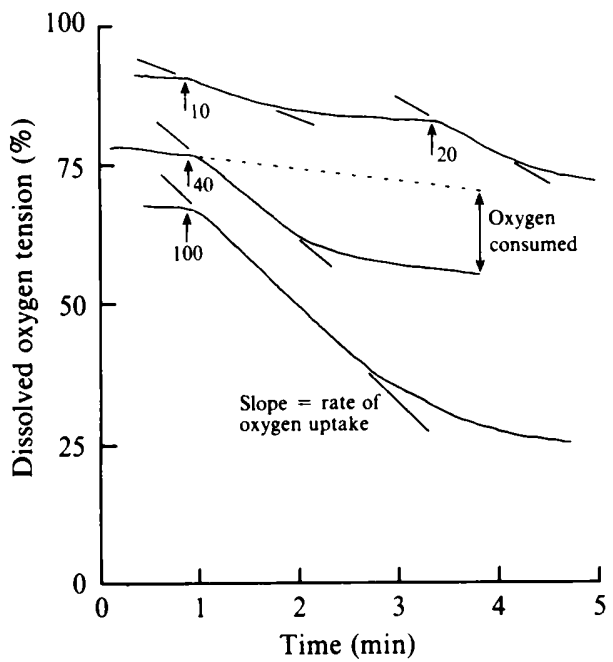

Fig. 1

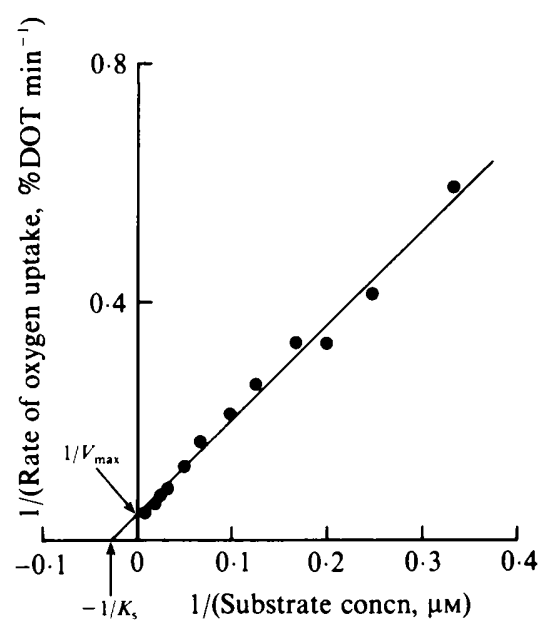

Fig. 2

Fig. 1. Redrawn chart record of oxygen uptake at different methylamine concentrations for obligate methylotroph B6/2. The arrows indicate addition of substrate and the adjacent number gives its final concentration $(\mu \mathrm{M})$ in the sample. The ordinate represents dissolved oxygen tension expressed as a percentage of the saturation value in equilibrium with air.

Fig. 2. An example plot of [1/(total rate of oxygen uptake - endogenous rate)] against [1/(substrate concentration)] for strain B6/2 supplied with different concentrations of methylamine. The culture was first grown on methylamine at a steady-state dilution rate of $0.240 \mathrm{~h}^{-1}$. The slope is $1.6 \mu \mathrm{mol} \mathrm{min}(\%$ dissolved oxygen tension) ${ }^{-1} \mathrm{I}^{-1}$ (correlation coefficient 0.994$) . \%$ DOT on the ordinate represents dissolved oxygen tension expressed as a percentage of the saturation value in equilibrium with air.

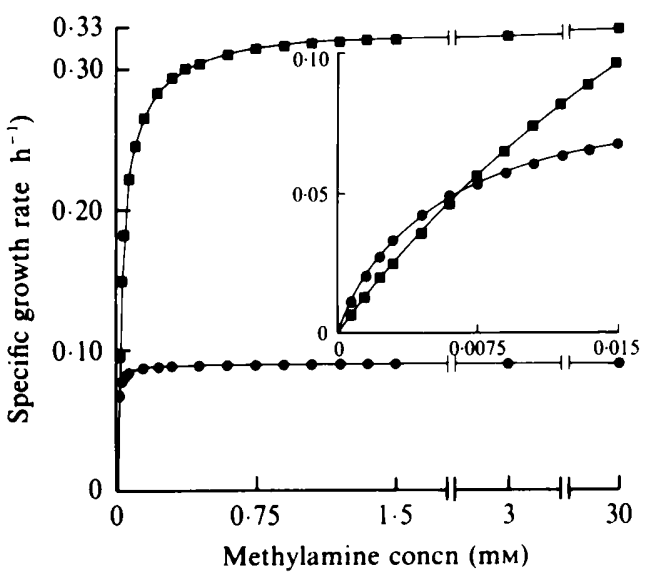

Fig. 3

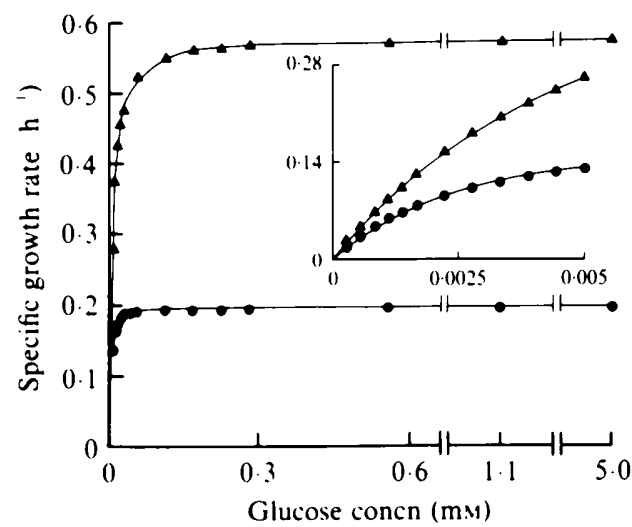

Fig. 4

Fig. 3. Plot of growth rate versus methylamine concentration for obligate methylotroph B6/2 ( $\square$ ) and facultative methylotroph DT26 (O), calculated by inserting experimentally determined $\mu_{\mathrm{m}}$ and $K_{\mathrm{s}}$ values into the Monod equation. The inset shows an enlargement of the region where methylamine concentration is less than $0.015 \mathrm{mM}$.

Fig. 4. Plot of growth rate versus glucose concentration for facultative methylotroph DT26 (O) and methazotroph NB4 $(\Lambda)$, calculated by inserting experimentally determined $\mu_{\mathrm{m}}$ and $K_{\mathrm{s}}$ values into the Monod equation. The inset shows an enlargement of the region where glucose concentration is less than $0.005 \mathrm{~mm}$. 


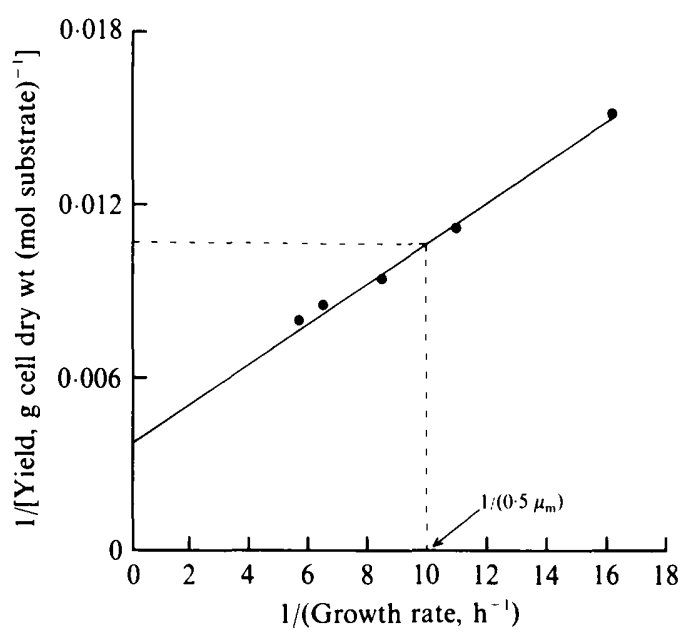

Fig. 5. An example plot of (1/yield) against (1/growth rate) for strain DT26 grown on glucose. The slope equals the maintenance coefficient, $0.7 \mathrm{mmol}$ glucose (g cell dry weight) ${ }^{-1} \mathrm{~h}^{-1}$ (correlation coefficient 0.996).

dry biomass $\mathrm{mol}^{-1}$ at $50 \%$ maximum growth rate) are considerably higher than experimentally determined values reported by Goldberg et al. (1976) but within the range of theoretical possibilities calculated by Anthony (1978). Goldberg et al. (1976) obtained yields of 9.3 to $11.4 \mathrm{~g} \mathrm{~mol}^{-1}$ on methylamine in continuous cultures at unstated relative growth rates for three Pseudomonas species known to use the serine pathway of $\mathrm{C}_{1}$-carbon assimilation. They reported higher yields of 15.7 to $17.3 \mathrm{~g} \mathrm{~mol}^{-1}$ on methanol for strains using the more efficient ribulose monophosphate assimilation pathway. The $C_{1}$-carbon assimilation pathways used by strains B6/2 and DT26 are not known, but since non-methane-using obligate methylotrophs generally have the ribulose monophosphate pathway (Anthony, 1982) it is likely that strain B6/2, at least, uses this pathway.

This work was supported by a contract for research from the Ministry of Agriculture, Fisheries and Food. The results are the properties of the Ministry and are Crown Copyright.

\section{REFERENCES}

ANTHONY, C. (1978). The prediction of growth yields in methylotrophs. Journal of General Microbiology 104, 91-104.

ANTHONY, C. (1982). The Biochemistry of Methylotrophs. London: Academic Press.

BiCKNELl, B. \& OWENS, J. D. (1980). Utilization of methyl amines as nitrogen sources by non-methylotrophs. Journal of General Microbiology 117, 89-96.

Goldberg, I., Rock, J. S., Ben-Bassat, A. \& MATELES, R. I. (1976). Bacterial yields on methanol, methylamine, formaldehyde and formate. Biotechnology and Bioengineering 18, 1657-1668.

HARRISON, D. E. F. (1973). Studies on the affinity of methanol- and methane-utilizing bacteria for their carbon substrates. Journal of Applied Bacteriology 36, 301-308.

Legan, J. D. \& OWENS, J. D. (1985). A note on the selection of a non-adhesive methylotrophic bacte- rium for use in continuous culture studies. Journal of Applied Bacteriology 58, 163-165.

Legan, J. D., Owens, J. D. \& Chilvers, G. A. (1987). Competition between specialist and generalist methylotrophic bacteria for an intermittent supply of methylamine. Journal of General Microbiology 133, 1061-1073.

NeIJSSEl, O. M. \& Tempest, D. W. (1976). Bioenergetic aspects of aerobic growth of Klebsiella aerogenes NCTC 418 organisms, growing in chemostat culture. Archives of Microbiology 107, 215-221.

PIRT, S. J. (1975). Principles of Microbe and Cell Cultivation. London: Blackwell.

PIRT, S. J. \& Callow, D. S. (1960). Studies of the growth of Penicillium chrysogenum in continuous flow culture with reference to penicillin production. Journal of Applied Bacteriology 23, 87-98. 